\title{
ALGUNOS COMENTARIOS SOBRE «EL PAPEL DEL PODER JUDICIAL EN LA TRANSICIÓN A LA DEMOCRACIA»
}

\author{
Juan Carlos Bayón \\ Universidad Autónoma de Madrid
}

RESUMEN. En este trabajo se discuten brevemente seis puntos a los que hace referencia Ernesto Garzón en «El papel del poder judicial en la transición a la democracia»: la imposibilidad de alcanzar una decisión acerca de los límites de la acción política si no es precisamente a través de la política; la discutible coherencia de aceptar el procedimiento democrático sólo para la esfera de los intereses y deseos secundarios; la idea de que la confiabilidad judicial depende de la coincidencia de las decisiones de los tribunales con el contenido más básico de la moral constitucional de la mayoría; la viabilidad y legitimidad de la promoción de la igualdad material y la homogeneidad económica a través de la protección jurisdiccional de los derechos sociales incorporados a las constituciones; el papel del poder judicial en relación con la justicia transicional; y la necesidad de distinguir la independencia judicial del activismo de los jueces.

Palabras clave: Constitucionalismo, derechos, democracia, derechos sociales, activismo judicial, justicia transicional.

ABSTRACT. In this paper six issues dealt with in Ernesto Garzón's «The Role of Judiciary in Transitions to Democracy» are cursorily discussed: the unavoidability of deciding on the limits of political activity precisely through politics; the dubious coherence of endorsing democratic procedures only in the realm of secondary interests and desires; the idea of judicial reliability as depending on the coincidence of judicial rulings with the deep commitments of the majority's constitutional morality; the feasibility and legitimacy of attaining equality and economic homogeneity through judicial enforcing of constitutionalised social rights; the role of judiciary relating to transitional justice; and judicial independence as distinguishable from judicial activism.

Keywords: Constitutionalism, rights, democracy, social rights, judicial activism, transitional justice. 
1 modo en que Ernesto GARZÓN VALDÉs ha contribuido a dar forma a nuestras reflexiones en torno al constitucionalismo, la relación entre democracia y derechos y el papel que en todo ello corresponde a los jueces ha sido sin la menor duda decisivo, incluso para quienes en cierta medida podamos discrepar de algunos de sus puntos de vista al respecto. En su trabajo «El papel del poder judicial en la transición a la democracia» ${ }^{1}$, que aquí se comenta, reaparecen sus ideas fundamentales y más conocidas sobre esas cuestiones, al tiempo que se introducen algunas tesis muy sugerentes relativas a los perfiles que adoptan en el contexto particular de los procesos de transición a la democracia. Como ocurre con toda obra importante - y la de GARZÓN VALDÉS sin duda lo es-, insistir en los puntos de acuerdo no sólo es innecesario, sino que le hace a uno correr el riesgo de decir peor lo que el autor comentado ha sabido expresar de una forma que no precisa de glosa alguna. Así que aunque sean muchos los aspectos en que su trabajo me parece iluminador, aquí me limitaré a presentar del modo más sucinto posible seis consideraciones críticas.

1. Una tesis central en la obra de GARZÓN VALDÉS, que ha defendido de hecho en múltiples ocasiones y ha adquirido una justa notoriedad, es la que sostiene que el ámbito de la decisión política - y el espacio en el que puede desplegarse justificadamente el procedimiento de decisión por mayoría- es sólo el de los intereses y deseos secundarios, esto es, el conjunto de cuestiones que no caen dentro del «coto vedado» (como él mismo dice en el texto que aquí se comenta, el coto vedado «fija, por exclusión, el ámbito de la decisión política» ${ }^{2}$ ). Debo confesar, sin embargo, que esta idea me ha suscitado siempre una perplejidad fundamental. No entiendo bien cómo cabe sostener que la determinación institucional de qué haya de considerar una comunidad como contenido de su coto vedado es algo que cae fuera del ámbito de la decisión política. Si en una sociedad en la que reina un desacuerdo profundo y persistente acerca de qué es lo justo ha de haber un «coto vedado», queda inexorablemente planteada la necesidad de alcanzar una decisión colectiva acerca de qué habrá de ser lo que en adelante no se va a admitir que se decida por mayoría. Y ello, se quiera o no, abre el interrogante de cómo alcanzar esa decisión. Dicho de otra manera: el trazado institucional del coto vedado no es ni puede ser un asunto que «cae fuera del ámbito de la política», puesto que, sea del modo que sea, tiene que someterse al poder de decisión de alguien. Y eso supone que ha de hacerse inevitablemente a través de la política. Naturalmente, no hay que dar por supuesto que el ámbito de la decisión política equivalga sin más al espacio en el que puede desplegarse justificadamente el procedimiento democrático: esto es, el hecho de que la decisión institucional acerca de los límites de la actividad política haya de adoptarse a través de la política - ¿cómo si no? - ciertamente no implica sin argumentos adicionales que haya de someterse precisamente al poder de decisión de la mayoría. Pero lo que no creo que pueda hacerse es defender la tesis de que la delimitación del contorno y contenido del coto vedado no ha de corresponderle a la mayoría como si fuese un simple corolario de la idea más básica de que ha de estar «sustraído a cualquier poder de decisión»³. Porque esto último, sencillamente, me parece imposible.

${ }^{1}$ GARZÓN VALDÉs [2002] 2003.

2 GARZÓn VAldÉs [2002] 2003, 133.

$3 \mathrm{Ni}$ creo tampoco que pueda afirmarse que «la determinación del contenido del "coto vedado" no puede ser función del Parlamento por razones conceptuales» (GARZÓN VALDÉS [2002] 2003: 134) sin acercarse pe- 
2. Hay un segundo punto que también me intriga en la posición de Ernesto GARZÓN. En mi opinión, la justificación de un procedimiento para la adopción de decisiones colectivas tiene que tomar en cuenta dos factores, entre los cuales ha de hacerse un balance: su valor intrínseco y su valor instrumental (es decir, la mayor o menor probabilidad de que conduzca a la adopción de decisiones justas, lo que depende a mi juicio de factores contextuales) ${ }^{4}$. Para justificar por qué el trazado del coto vedado no debería someterse al poder de decisión de la mayoría puede sostenerse - y en efecto se ha sostenido- que el procedimiento democrático carece en realidad de valor intrínseco (una posición, por cierto, que yo no comparto); y también que su valor instrumental, en lo que concierne al reconocimiento de derechos fundamentales, es sistemática y no contingentemente menor que el de los mecanismos contramayoritarios típicos del constitucionalismo «fuerte» o clásico (una idea que suele defenderse sobre la base de argumentos que tampoco me parecen convincentes) ${ }^{5}$. Pero lo que me interesa destacar ahora es que todo el que razone de alguna de estas maneras debería explicar - y creo que también es pertinente dirigirle la pregunta a Ernesto GARZÓN-por qué razón acepta entonces como justificado el procedimiento de decisión por mayorías - y no algún otro- en el «ámbito de los intereses y deseos secundarios». No creo que resultasen vías muy prometedoras responder que sólo en este ámbito posee valor intrínseco, o que sólo en este ámbito su valor instrumental es sistemáticamente superior a otros procedimientos de decisión alternativos ${ }^{6}$. Pero si no se contesta de alguno de esos modos me parece que queda sin una justificación clara la apuesta por el procedimiento democrático en las cuestiones que no involucran derechos (y sólo en ellas).

3. Un tercer punto que me gustaría destacar es la idea de GARZÓN VALDÉS de que la legitimidad de los tribunales constitucionales debe medirse en términos de «confiabilidad judicial», que a su juicio «sólo se da si existe, por lo general, coincidencia entre la interpretación [...] del tribunal y la interpretación que sustenta la communis opinio», pero puntualizando, eso sí, que «en algunos casos esta coincidencia puede producirse sólo después de que el tribunal [...] ha explicitado las razones de su interpretación» ${ }^{7}$. Si entiendo bien, la idea vendría a ser que lo que legitima la interpretación del tribunal

ligrosamente a una petición de principio. Por otra parte, concluir que «[s]on los padres de la Constitución quienes han de fijar el contenido del "coto vedado"» (GARZÓN VALDÉS [2002] 2003: 133) deja sin responder la cuestión decisiva de quiénes y con qué título habrían de actuar como tales (y cómo, de no haber unanimidad, tendrían que alcanzarse las decisiones entre ellos).

${ }^{4}$ Para un desarrollo pormenorizado de un planteamiento de este tipo, vid. BAYÓN 2004; y WALDRON 2006 (donde revisa la posición que sostuvo anteriormente).

5 Vid. en MCGANN 2004 una ingeniosa explicación del modo en que, en contra de estereotipos muy difundidos, dadas ciertas condiciones el mecanismo de toma de decisiones colectivas que mejor protege a las minorías puede ser precisamente la regla de la mayoría (y no mayorías reforzadas como las que suelen requerirse en los Estados constitucionales modernos para la reforma de la Constitución).

${ }^{6}$ No me parece que pueda sostenerse, por ejemplo, que «bajo el velo de la ignorancia» un sujeto racional elegiría el procedimiento mayoritario para la toma de decisiones en el ámbito de los intereses y deseos secundarios (porque a la larga maximizaría la probabilidad de satisfacción de los deseos propios), pero no para la adopción de las que conciernen a los bienes básicos protegidos en el coto vedado (porque su importancia para cada individuo es tal que ponerlos en manos de la mayoría sería un riesgo excesivamente alto). Ninguna de esas ideas me parece convincente: en cuanto a la segunda, basta con repetir que algún procedimiento de decisión al respecto es inevitable y no está probado que, en cualquier contexto, el procedimiento democrático represente una amenaza mayor para aquellos bienes básicos que los clásicos procedimientos contramayoritarios del constitucionalismo; y en cuanto a la primera, como se ha subrayado muchas veces, el argumento ignoraría la insensibilidad del procedimiento mayoritario hacia la intensidad de las preferencias.

7 GARZÓN VALDÉS [2002] 2003: 132. 
—y le confiere «confiabilidad»— sería la coincidencia no con los juicios superficiales (inmediatos, o no meditados) de la mayoría acerca del punto debatido, sino con el resultado de haber puesto en equilibrio reflexivo los compromisos más profundos de la moral constitucional de la mayoría (haciéndole ver así en algunos casos a dicha mayoría, argumentativamente, que sus juicios superficiales eran erróneos en términos de sus propios compromisos fundamentales). Y esa clase de «esclarecimiento» requeriría por definición un procedimiento de decisión de naturaleza no agregativa, sino estrictamente deliberativa o argumentativa. Ésta me parece una idea sumamente interesante ${ }^{8}$. El problema es que en el diseño del constitucionalismo clásico no hay un mecanismo institucionalizado de feed-back que, si tal es el caso, permita ponerle de manifiesto al tribunal que esa clase de coincidencia, en contra de lo que él cree, no se ha producido?. En ese sentido, mecanismos de «constitucionalismo débil» (como los de Canadá o el Reino Unido) pueden permitir el ajuste o corrección recíproca entre jueces y legisladores — aumentando así la calidad deliberativa del proceso democrático-, pero reservando la última palabra a aquél cuya «confiabilidad» (reliability) se calibra finalmente a través de los mecanismos inexorables de la rendición de cuentas inherente a la responsabilidad democrática (accountability).

4. Un cuarto punto, relativo ya al papel de los jueces constitucionales en el supuesto específico de las transiciones a la democracia, viene suscitado por la afirmación de GARZÓN VALDÉS de que su actividad puede y debe contribuir a la igualdad material, esto es, al establecimiento de una sociedad económicamente homogénea ${ }^{10}$, lo que sería - y en eso, por descontado, estoy de acuerdo — una condición necesaria para el afianzamiento de la democracia. Pero yo no estoy tan seguro de que realmente puedan y, en la medida en que puedan, deban hacerlo. Los derechos sociales que muchas constituciones reconocen no forman una categoría unitaria desde el punto de vista estructural ${ }^{11}$. Y aquellos que por su estructura no presentan problemas particulares en cuanto a la posibilidad de su protección jurisdiccional son, según creo, los que menos incidencia pueden tener en el «establecimiento - efectivo- de una sociedad económicamente homogénea». En cambio, los que sí podrían tenerla, requerirían para su satisfacción decisiones de política económica que es más que discutible que los jueces deban adoptar, tanto por cuestiones de competencia técnica como, sobre todo, de legitimidad ${ }^{12}$. Creo que algo similar a la idea de Lawrence SAGER de infra-aplicación de la constitución por los jueces sería aquí pertinente ${ }^{13}$. Y todo ello sin que tampoco convenga olvidar, por cierto, cómo en contextos de transición la invocación de derechos fundamentales por parte de los jueces constitucionales ha servido en una serie de casos, paradójicamente,

${ }^{8}$ Y muy similar, por cierto, a la que recientemente ha articulado en detalle WALUCHOW, 2007, cap. 6.

9 Éste es, de hecho, el reproche central de Andrei MARMOR a la posición de WALUCHOW: cfr. MARMOR 2007: 90-92.

10 GARZÓN VALDÉs [2002] 2003: 139-141.

${ }^{11}$ Cfr. LAPORTA 2004, 298 ss.; y CruZ PARCERO 2007, 96-99.

${ }_{12}$ Vid. LAPORTA 2004: 309-317, donde se muestran de manera convincente las distintas posibilidades y dificultades de la protección jurisdiccional de los derechos sociales según se trate, por una parte, de derechos que se configuran estructuralmente como libertades o que tienen que ver con demandas para cuya satisfacción ya existe un entramado institucional previo o, por otra parte, de aquellos que estructuralmente son derechos de prestación de recursos, bienes o servicios, o a la producción de bienes públicos todavía inexistentes.

${ }_{13}$ Cfr. SAGER [2004] 2007: 103-106, 117, 139. 
para invalidar decisiones del nuevo legislador democrático encaminadas justamente al «establecimiento de una sociedad económicamente más homogénea» ${ }^{14}$.

5. Un quinto punto, que GARZÓN no menciona expresamente en este trabajo, pero del que se ha ocupado en otras ocasiones ${ }^{15}$ y que sin duda es muy relevante a la hora de reflexionar sobre «el papel del poder judicial en las transiciones a la democracia», es el que tiene que ver, justamente, con los problemas de la llamada «justicia transicional», esto es, con la exigencia de responsabilidades por las violaciones de derechos fundamentales llevadas a cabo durante el régimen anterior. Si se acepta que muchas veces lo más característico de esos períodos es precisamente el carácter contraproducente de intentar cumplir las exigencias de la justicia en condiciones ideales (porque ello podría poner en peligro el proceso mismo de democratización) y la necesidad de perseguir en su lugar un second-best apropiado para las circunstancias particulares de cada proceso de cambio ${ }^{16}$, de lo que resultan con frecuencia compromisos sumamente delicados, parece preferible que esos compromisos se convaliden - o no- a través de las urnas y que los jueces constitucionales muestren una actitud al respecto de autocontención y deferencia hacia el legislador. Las alternativas serían que los jueces hicierar valer de manera incondicionada las exigencias ideales de justicia (aprovechando que probablemente estarán plasmadas en abstracto como derechos constitucionales) o que sustituyeran al legislador en su apreciación del modo en que debería articularse ese compromiso o second-best apropiado a las circunstancias reales: y ambas cosas, de distintas maneras, podrían ser formas de hacer un flaco servicio a la causa de la transición hacia la democracia.

6. Por último, GARZÓN VALDÉS tiene sin la menor duda toda la razón al apuntar que un poder judicial independiente es un instrumento imprescindible para combatir el abuso o la corrupción de los poderes. Pero a mi modo de ver la independencia del poder judicial debe plasmarse en el respeto al principio de legalidad, la promoción de

${ }^{14}$ Como ha sucedido en algunas ocasiones en los recientes procesos de transición a la democracia de países de la Europa central y del este. Así, por ejemplo, en Polonia el Tribunal Constitucional invalidó una ley que establecía límites a la posibilidad de revisión de la renta de determinados arrendamientos urbanos alegando que ello vulneraba el derecho de propiedad (GARLICKI, 2002: 277); en Eslovenia, donde el legislador había excluido de los procesos de reprivatización de tierras las grandes fincas «de origen feudal», el Tribunal Constitucional exceptuó las que hubieran sido propiedad de las Iglesias «en atención al papel que la Constitución les reconoce como instituciones de interés general» (SADURSKI, 2005: 103); y en Bulgaria el Tribunal Constitucional invalidó una ley que condicionaba el disfrute de una pensión a la no percepción de otros ingresos por encima de un determinado monto alegando que ello limitaba inaceptablemente o el derecho a trabajar o el derecho de propiedad (SADURSKI, 2005: 193). Sobre el escaso papel que en general han desempeñado los tribunales en cuanto a la promoción de la igualdad material en los procesos de transición y cómo, por el contrario, han servido a veces para preservar intereses de grupos privilegiados que justamente podían verse amenazados por el funcionamiento de los incipientes mecanismos democráticos, vid. HiRSCHL 2004, especialmente, 12-15 y 216-217.

15 Cfr., p. ej., GARZÓN VALDÉs [1999] 2004.

${ }^{16}$ Un punto de vista con el que simpatizo y que sostiene, por ejemplo, TEITEL, 2000: 224 y 227. Sobre la noción de «ideal regulativo» y la inconveniencia de dar por supuesto que en cualesquiera circunstancias es mejor aquel curso de acción que intenta producir el estado de cosas más cercano a dicho ideal, vid. MARTí 2005. Ernesto GARZÓN, en cambio, se ha pronunciado en alguna ocasión en contra de la admisibilidad de compromisos como los conducentes tan sólo a alguna forma de castigo selectivo (cfr. GARZÓN VALDÉs [1999] 2004: 345 347), aunque no me resulta claro si su postura va referida únicamente a procesos de transición de los que se piensa que las razones para conformarse tan sólo con ese second-best no son reales - y si lo son o no es algo que dependerá siempre de un juicio empírico sobre las circunstancias particulares de cada caso-, o habría de tener por el contrario alcance general. 
la seguridad jurídica y la imparcialidad en la aplicación de la ley ${ }^{17}$. A eso (nada más, pero tampoco nada menos) creo que debería ceñirse en realidad «el papel del poder judicial en la transición a la democracia». Me parece, por el contrario, que actuar como «promotores de la igualdad material» —o hacer prevalecer su criterio sobre las delicadas decisiones que puede requerir la «justicia transicional»—- tiene que ver más con el activismo que con la independencia judicial. Y el activismo judicial sólo a veces favorece la causa de los derechos y siempre, en cambio, daña la de la democracia.

\section{REFERENCIAS BIBLIOGRÁFICAS}

BAYÓN, J. C., 2004: «Democracia y derechos: problemas de fundamentación del constitucionalismo», en J. BETEGÓN et al. (eds.), 2004: Constitución y derechos fundamentales, Madrid: Centro de Estudios Políticos y Constitucionales, pp. 67-138.

Cruz Parcero, J. A. (2007): El lenguaje de los derechos. Ensayo para una teoría estructural de los derechos, Madrid: Trotta, 2007.

GARLICKI, L.L., 2002: «The Experience of the Polish Constitutional Court», en W. SADURSKI, ed., 2002: Constitutional Justice, East and West. Democratic Legitimacy and Constitutional Courts in Post-Communist Europe in a Comparative Perspective, The Hague: Kluwer Law Internationa, pp. 265-282.

GARZÓN VAldÉs, E. [1999] 2004: «Dictatorship and Punishment: A Reply to Scanlon and Teitel», en H. Hongu Kon y R. C. Stye (eds.), 1999: Deliberative Democracy and Human Rights, New Haven: Yale University Press, pp. 291-300; hay trad. cast. de P. BERGALLO y M. ALEGRE, «Dictadura y castigo: una réplica a Scanlon y Teitel», en H. Hongju KoH y R. C. SLYE (eds.) 2004: Democracia deliberativa y derechos humanos, Barcelona: Gedisa, pp. 343 352 (por donde se cita).

- [2002] 2003: «El papel del poder judicial en la transición a la democracia», Jueces para la Democracia, 45, 2002, pp. 45-52; publicado también en Isonomía, 18, 2003, pp. 27-46; y en J. MALEM, J. OROZCO y R. VÁZQUEZ (eds.) 2003: La función judicial. Ética y democracia, Barcelona: Gedisa, pp. 129-145 (por donde se cita).

Hierro, L., 1998: Estado de Derecho. Problemas actuales, México: Fontamara, 1998.

HIRSCHL, R., 2004: Towards Juristocracy. The Origins and Consequences of the New Constitutionalism, Cambridge-Mass.: Harvard University Pres.

LAPORTA, F. J., 2004: «Los derechos sociales y su protección jurídica: Introducción al problema», en J. BETEGÓN et al. (eds.) 2004, Constitución y derechos fundamentales, Madrid: Centro de Estudios Políticos y Constitucionales, pp. 297-325.

MARMOR, A., 2007: «Are Constitutions Legitimate?», Canadian Journal of Law and Jurisprudence, 20, 69-94.

MARTí, J. L., 2005: «La nozione di ideale regolativo: note preliminari per una teoria degli ideali regolativi nel diritto», Ragion Pratica, 25, pp. 381-404.

McGann, A. J., 2004: «The Tyranny of the Supermajority: How Majority Rule Protects Minorities», Journal of Theoretical Politics, 16, pp. 53-77.

SAduRSKI, W., 2005: Rights Before Courts. Study of Constitutional Courts in Postcommunist States of Central and Eastern Europe, Dordrecht: Springer.

SAgER, L. G. [2004] 2007: Justice in Plainclothes. A Theory of American Constitutional Practice, New Haven: Yale University Press; hay trad. cast. de A. TORRES PÉREZ, con estudio prelimi-

${ }^{17}$ Cfr. Hierro, 1998: 45-57. 
nar de V. FERRERes COMElla, Juez y democracia. Una teoría de la práctica constitucional norteamericana, Madrid/Barcelona: Marcial Pons (por donde se cita).

Teitel, R., 2000: Transitional Justice, Oxford: Oxford University Press.

WALDron, J., 2006: «The Core of the Case Against Judicial Review», Yale Law Journal, 115, pp. 1346-1406.

Waluchow, W., 2007: A Common Law Theory of Judicial Review: The Living Tree, Cambridge/New York: Cambridge University PreSs. 